\title{
Practice Patterns, Beliefs, and Perceived Barriers to Care Regarding Dementia: A Report from the American Academy of Family Physicians (AAFP) National Research Network
}

\author{
Thomas V. Stewart, MA, Natalia Loskutova, MD, PhD, James M. Galliber, PhD, \\ Gregg A. Warshaw, MD, Letoynia J. Coombs, EdD, Elizabeth W. Staton, MSTC, \\ Jessica M. Huff, MS, MPH, and Wilson D. Pace, MD
}

Purpose: Given the increasing age of the US population, understanding how primary care is delivered surrounding dementia and physicians' perceived barriers and needs associated with this care is essential.

Methods: A 29-item questionnaire was developed by project investigators and family physician consultants and mailed to a random sample of 1500 US members of the American Academy of Family Physicians in 2008; 2 follow-up mailings were sent to nonrespondents. Physicians were queried about sociodemographic characteristics, practice patterns, and beliefs (including challenges, barriers, and needs) about care processes focusing on dementia among older patients.

Results: The response rate was $60 \%$, with respondents statistically comparable $(P>.05)$ to the American Academy of Family Physicians physician population. Among physicians, 93\% screen and/or conduct diagnostic evaluations for dementia in older patients, whereas $91 \%$ provide ongoing primary care for patients with dementia whether or not they screen for or diagnose dementia. Forty percent of physicians refer some patients with suspected dementia to other providers (primarily neurologists) to verify diagnosis, for comanagement, or both. Factors affecting the diagnosis of dementia and the delivery of dementia care included patient behavior challenges (aggressiveness, restlessness, paranoia, wandering); comorbidities (falls, delirium, adverse medication reactions, urinary incontinence); caregiver challenges (fatigue, planning for patient's institutional placement, anger); and structural barriers (clinician time, time required for screening, limited treatment options). Tools needed to provide enhanced dementia care included better assessment tools, community resources, and diagnostic and screening tools.

Conclusion: Family physicians are highly involved in the assessment and routine care of patients with suspected dementia or diagnosed with dementia, although a relative few are not. This is despite the recognized challenges physicians encounter in the assessment and care processes. ( $\mathrm{J}$ Am Board Fam Med 2014;27:275-283.)

Keywords: Dementia, Practice-based Research, Primary Health Care

Dementia is a general term describing a serious loss of at least 2 cognitive functions, such as memory, attention, thinking, or language, caused by a num-

This article was externally peer reviewed.

Submitted 17 October 2012; revised 7 November 2013; accepted 11 November 2013.

From the AAFP National Research Network, Leawood, KS (TVA, NL, JMG, EWS, WDP); the Department of Sociology, University of Missouri, Kansas City (JMG); the Department of Family and Community Medicine, University of Cincinnati, Cincinnati, OH (GAW); the Department of Family Medicine, University of Colorado Denver, Aurora (LJC, EWS, WDP); and the Department of Medical Education and Research, Danbury Hospital, Danbury, CT (JMH). ber of brain disorders. Alzheimer's disease (AD), which accounts for $60 \%$ to $80 \%$ of all dementia cases, is the most common cause. ${ }^{1}$ About $13.9 \%$ of

Funding: This study was funded by an unrestricted research grant from Forest Laboratories, Inc.

Prior presentation: This study was presented as a poster at the Annual Conference of the North American Primary Care Research Group (NAPCRG) in Rio Grande, Puerto Rico (November 16, 2008).

Conflict of interest: none declared.

Corresponding author: Thomas V. Stewart, MA, National Research Network, American Academy of Family Physicians, 11400 Tomahawk Creek Parkway, Leawood, KS 66211 (E-mail: tstewart@aafp.org). 
older US adults (about 40 million people $>65$ years old) and $44 \%$ of US adults $>85$ years old have dementia. ${ }^{2}$ The burden of suffering from dementia is great for patients, their families, and the larger community because of increasing personal dependency and depression as well as family/caregiver stress, anxiety, and depression. Financial stress is also significant for the large percentage of patients who eventually require nursing home placement. Medical, long-term, and end-of-life care costs per patient are estimated to be, on average, $\$ 78,000$ a year for a person with dementia and $\$ 13,000$ a year for a person of the same age without dementia, totaling to a cost of $\$ 215$ billion for all people with dementia. ${ }^{3,4}$ The strongest risk factor for the development of dementia is age. A major challenge for patients, caregivers, and clinicians is to recognize changes in memory, thinking, and other cognitive functions and to distinguish normal aging from early dementia. This is particularly challenging in primary care, where the majority of dementia patients (40\% to $80 \%)$ go undiagnosed until later stages. $^{5-7}$

The reasons for this delay are complex: clinicians may not recognize early symptoms; patients, clinicians, and families may deny or fail to recognize cognitive concerns as part of the dementia process; the public and clinicians may believe that cognitive impairment is normal in aging; and interventions by caregivers may unintentionally mask the recognition of cognitive decline. ${ }^{8}$ Moreover, it is unclear whether primary care clinicians consider late recognition an issue that should be addressed. Nonetheless, the major reason for the large numbers of patients $>65$ years old with undiagnosed dementia is the difficulty in making the diagnosis, especially in its early stages. ${ }^{9}$

With the aging of the population, the percent age of the population in North America likely to be diagnosed with dementia is estimated to increase by $49 \%$ by 2020 and $172 \%$ by $2040 .{ }^{10}$ Primary care physicians must learn to care for these patients; thus, it is useful to understand family physicians' current clinical expectations, actions, and needs related to dementia care.

The American Academy of Family Physicians (AAFP) National Research Network (NRN) surveyed a representative sample of active AAFP members residing in the United States regarding their practice patterns related to screening, diagnosing, referring, and caring for patients with de- mentia; dementia-related behaviors, complications, comorbidities, and family/caregiver problems often associated with dementia; and perceived barriers to diagnosing and caring for patients with dementia.

\section{Methods}

An initial draft questionnaire consisting of approximately 50 items was developed in an iterative fashion by the study investigators and consultants in the field of geriatrics. This draft survey then was sent to selected physician members of the AAFP NRN who had agreed to review study materials. Sixteen members reviewed the survey, after which the original questionnaire was reduced to 29 items and approved by the AAFP Institutional Review Board in March 2008.

We selected a random sample from the AAFP's master membership database $(\mathrm{N}=60,129)$ for December 31, 2007. AAFP physicians who spent at least $50 \%$ time in direct patient care and reported a mailing address within the 50 United States or the District of Columbia were considered for the survey. From this eligible population of 30,174 members, we selected a random sample of 1,500 using a computerized random sampling strategy. A final sample was randomly selected from a cluster of members based on the randomly generated 7-digit AAFP identifier given to each member. We determined the number of 1,500 was large enough by conventional standards to adequately describe this population within an acceptable margin of error for both 5 -point Likert items (95\% $\pm 0.12 \%$; estimated variance of 0.20$)$, and dichotomous survey items ( $95 \% \pm 5.0 \%$; estimated $P=.50)$, both assuming a $50 \%$ response rate, which was exceeded (final response rate, 60\%). In June 2008 we mailed the final survey, along with a $\$ 2.00$ bill, to the sampled physicians. Two follow-up mailings, each approximately 30 days after the preceding mailing, were sent to nonrespondents. The $\$ 2.00$ incentive also was included in the second follow-up mailing.

\section{Survey Items}

We designed the survey to investigate the following components of the care process for patients with dementia in primary care settings: screening; diagnostic evaluation; referral; perceived barriers associated with the treatment and management of dementia; dementia-related behaviors, complications, and comorbidities; family and caregiver problems; 
and the perceived need for better tools to diagnose/ treat dementia (survey instrument is available on request). Some aspects of this multifaceted process were represented by a single dichotomous (yes/no) item ("Do you screen?"), whereas others included multiple-response items in which each response was treated as dichotomous (yes/no) and/or treated as a multiple-count item (eg, "perceived barriers to diagnosing and caring for patients with dementia," "challenging family/caregiver problems associated with dementia").

We considered 3 outcomes for this report: (1) physician ordering of brain imaging as part of screening and/or diagnostic evaluation for dementia (yes/ no), (2) assessment of patients who screened positive using more sensitive tests (yes/no), and (3) referral of patients with suspected dementia to other specialists for treatment (yes/no). Other dichotomous outcome items were not selected for further analysis or reporting because of their relative lack of variability. Full results are available on request. The 9 independent variables from the survey instrument were physician respondent's age group and sex; percentage of patient population $\geq 65$ years old; geographic location of practice (urban, suburban, rural); perceived comorbidities, behaviors/complications, family/caregiver problems associated with dementia; number of tools needed to better diagnose and treat dementia; and the number of perceived practice-related barriers to diagnosing and treating patients with dementia. We also included 2 items from the AAFP Master Membership Database: years since medical school graduation and percentage of time spent in direct patient care.

\section{Statistical Analysis}

This study was not designed for hypothesis testing, and therefore most analyses conducted were descriptive. Preliminary analysis included univariate and bivariate techniques for the 3 outcome items and independent variables. We used $\chi^{2}$, Pearson correlation, and $t$ tests to assess possible bivariate associations between the named outcomes and each of the independent variables. Those items found to be statistically associated with a given dependent variable $(P \leq .20)$ then were entered simultaneously into a multivariate logistic regression analysis for each outcome with the other items excluded. We used SPSS software version 17.0.1 (IBM/SPSS Inc., Chicago, IL) and SAS software version 9.3 (SAS Inc., Cary, NC) for the analyses and included summary descriptions of demographic data and frequency data of survey answers.

\section{Results}

\section{Data Collection}

Eighteen questionnaires (1\%) were returned with incorrect addresses, incomplete, or the respondent indicated that they had retired from practice, leaving a valid initial sample of 1482 . There were 885 surveys $(60 \%)$ returned; 34 of these respondents (4\%) stated that they did not see patients $>65$ years old and were eliminated from the analysis, leaving 851 completed surveys for analysis. These 851 physicians estimated their patients $>65$ years old (relative to their total patient population) to be between $1 \%$ and $20 \%$ ( $45 \%$ of respondents), $21 \%$ and $40 \%$ (37\% of respondents), and $\geq 41 \%$ ( $18 \%$ of respondents).

Table 1 summarizes selected demographic characteristics of respondents. We compared their characteristics to those of the larger AAFP population from which the sample was selected to assess the representativeness of the respondents relative to the overall population. Sampled respondents did not differ statistically $(P>.05)$ from the larger population on 5 of 6 comparisons. For time spent in direct patient care, however, the 2 means (90\% and 89\%) were significantly different $(P=.02)$ because of the relatively large sample size $(\mathrm{n}=851)$, although this is not likely to be clinically meaningful.

\section{Descriptive Analysis}

\section{Screening and Diagnostic Evaluation}

Fifty-six percent of physicians indicated that they screen asymptomatic individuals "at least some of the time" (Table 2). Age, family history, and cerebrovascular risk factors often trigger the decision to screen. When conducting screening, 80\% reported using a specific screening tool, with those most frequently mentioned being the Mini-Mental Status Examination (Psychological Assessment Resources, Inc., Lutz, FL) and the clock-drawing test. No other screening test was mentioned by $>7 \%$ of respondents.

An overwhelming majority of the respondents (90\%) conduct diagnostic evaluations for dementia in patients based on early signs or symptoms or patient and/or caregiver concerns of dementia. On the basis of physician reports, $52 \%$ both screen and conduct diagnostic evaluations, $37 \%$ only conduct diagnostic evaluations, $4 \%$ only screen, and $7 \%$ do neither. 
Table 1. Selected Sociodemographic Characteristics of Survey Respondents Compared to American Academy of Family Physicians (AAFP) Population (Overall Sample)

\begin{tabular}{|c|c|c|c|c|c|}
\hline \multirow[b]{2}{*}{ Sampled Respondent Characteristics } & \multirow[b]{2}{*}{ Respondents (n) } & \multirow{2}{*}{$\begin{array}{l}\text { Respondents Compared to } \\
\text { Overall Sample }\end{array}$} & \multicolumn{3}{|c|}{ Test Results } \\
\hline & & & $x^{2}$ & $\mathrm{df}$ & $P$ \\
\hline Clinician age (years) $(\mathrm{n}=848$ respondents) & & & 3.12 & 3 & .37 \\
\hline$<40$ & 119 & $14 \%$ vs. $15 \%$ & & & \\
\hline $40-49$ & 271 & $32 \%$ vs. $35 \%$ & & & \\
\hline $50-59$ & 331 & $39 \%$ vs. $37 \%$ & & & \\
\hline$\geq 60$ & 127 & $15 \%$ vs. $13 \%$ & & & \\
\hline Sex $(n=851$ respondents $)$ & & & 0.23 & 1 & \\
\hline Female & 255 & $30 \%$ vs. $31 \%$ & & & \\
\hline Male & 596 & $70 \%$ vs. $69 \%$ & & & \\
\hline $\begin{array}{l}\text { Time since medical school graduation } \\
\text { (years) }(\mathrm{n}=851 \text { respondents) }\end{array}$ & & & 6.69 & 3 & .08 \\
\hline $0-10$ & 68 & $8 \%$ vs. $9 \%$ & & & \\
\hline $11-20$ & 289 & $34 \%$ vs. $40 \%$ & & & \\
\hline $21-30$ & 315 & $37 \%$ vs. $34 \%$ & & & \\
\hline$\geq 31$ & 179 & $21 \%$ vs. $18 \%$ & & & \\
\hline $\begin{array}{l}\text { Practice location (by Census region) } \\
\quad(\mathrm{n}=843 \text { respondents })\end{array}$ & & & 0.31 & 2 & .86 \\
\hline Northeast & 128 & $15 \%$ vs. $15 \%$ & & & \\
\hline Midwest & 238 & $28 \%$ vs. $28 \%$ & & & \\
\hline South & 278 & $33 \%$ vs. $34 \%$ & & & \\
\hline West & 196 & $23 \%$ vs. $22 \%$ & & & \\
\hline Practice location ( $\mathrm{n}=843$ respondents) & & & 0.31 & 2 & .86 \\
\hline Urban & 169 & $20 \%$ vs. $21 \%$ & & & \\
\hline Suburban & 379 & $45 \%$ vs. $44 \%$ & & & \\
\hline Rural & 295 & $35 \%$ vs. $36 \%$ & & & \\
\hline Percentage of time spent in direct patient care & 851 & $90 \%$ vs. $89 \%$ & $Z=2.43$ & & .02 \\
\hline
\end{tabular}

Respondents who reported screening and/or conducting diagnostic evaluations $(\mathrm{n}=794)$ provided information about follow-up after a positive screen or as part of diagnostic evaluation of dementia in patients (Table 2). The collection of further history from the patient/family, additional cognitive testing, routine laboratory work, and brain imaging are commonly part of the diagnostic evaluation. The overwhelming reason $(>98 \%)$ given for ordering lab work or brain imaging was to rule out other causes of dementia.

\section{Caring for Patients With Dementia}

Of the 851 eligible respondents, 771 (91\%) stated that they provide routine ongoing primary care for patients with dementia, regardless of whether they screen and/or conduct clinical evaluations for dementia (Table 3). Furthermore, of the 57 physicians $(7 \%)$ who neither screen nor conduct clinical evaluations for dementia, 51\% nonetheless provide primary care for such patients.
Respondents who routinely provide primary care for patients with dementia $(\mathrm{n}=771)$ are most challenged by dementia-related behaviors and complications, including aggressiveness, restlessness/agitation, paranoia, wandering, and sundowning. They also indicated that the most challenging comorbidities to manage were falls, delirium, adverse reactions to medications, and urinary incontinence. When asked about family/caregiver problems associated with dementia that were challenging to manage, they reported fatigue/exhaustion, planning for patient's institutional placement, and anger.

\section{Barriers and Tools Needed to Care for Patients With \\ Dementia}

Respondents were asked about perceived barriers to diagnosing and caring for patients with dementia. Their most frequent responses included inadequate clinician time (78\%), length of time needed to administer screening tools $(51 \%)$, and limited treatment options (48\%). They also indicated that the tools they 
Table 2. Physician Responses to Survey Items Related to Screening and Diagnostic Evaluation for Dementia

\begin{tabular}{|c|c|}
\hline Screening/Diagnostic Evaluation Item & $\begin{array}{l}\text { "Yes" Responses, } \\
\text { n (\%) }\end{array}$ \\
\hline $\begin{array}{l}\text { "Do you ever screen for dementia in } \\
\text { asymptomatic adults?" }(\mathrm{n}=851 \\
\text { were asked) }\end{array}$ & $476(56)$ \\
\hline \multicolumn{2}{|l|}{$\begin{array}{l}\text { Criteria used as decision rules for screening } \\
\qquad(\mathrm{n}=476 \text { responded })\end{array}$} \\
\hline Age & $363(76)$ \\
\hline Family history & $344(72)$ \\
\hline Other cerebrovascular risk factors & $341(72)$ \\
\hline Sex & $56(12)$ \\
\hline Use specific tools to screen $(n=476)$ & $379(80)$ \\
\hline Mini-mental status exam (379) & $359(95)$ \\
\hline Clock drawing test (379) & $216(57)$ \\
\hline $\begin{array}{l}\text { Short portable mental status exam } \\
\quad(379)\end{array}$ & $26(7)$ \\
\hline $\begin{array}{l}\text { "Do you conduct diagnostic evaluations } \\
\text { based on early signs or symptoms, } \\
\text { patient concerns, or caregiver } \\
\text { concerns?" ( } \mathrm{n}=851 \text { were asked) }\end{array}$ & $762(90)$ \\
\hline \multicolumn{2}{|l|}{$\begin{array}{l}\text { Screening and diagnostic evaluation status } \\
\qquad(\mathrm{n}=851 \text { responded })\end{array}$} \\
\hline Doctor does both & $444(52)$ \\
\hline $\begin{array}{l}\text { Doctor conducts diagnostic evaluations } \\
\text { only }\end{array}$ & $318(37)$ \\
\hline Doctor screens only & $32(4)$ \\
\hline Doctor does neither & $57(7)$ \\
\hline $\begin{array}{l}\text { "If you obtain a positive screen or suspect } \\
\text { dementia in a patient, do you } \\
\text { follow-up with a more sensitive test } \\
\text { in your office for presence of } \\
\text { dementia?" ( } \mathrm{n}=794 \text { were asked) }\end{array}$ & $579(73)$ \\
\hline $\begin{array}{l}\text { "Do you routinely collect patient history as } \\
\text { part of the diagnostic process?" } \\
(\mathrm{n}=794 \text { were asked })\end{array}$ & $758(95)$ \\
\hline $\begin{array}{l}\text { "When you assess patient, do you routinely } \\
\text { order lab work?" (n = } 794 \text { were } \\
\text { asked) }\end{array}$ & $739(93)$ \\
\hline $\begin{array}{l}\text { "When you diagnose a patient, do you } \\
\text { routinely order brain imaging?" } \\
(\mathrm{n}=794 \text { were asked) }\end{array}$ & $527(66)$ \\
\hline
\end{tabular}

needed to better diagnose and/or treat patients with dementia included better assessment tools (62\%), access to community resources (49\%), diagnostic tools (48\%), and screening tools (40\%).

\section{Referral to Other Clinicians}

Of the 823 physicians who reported that they screen, diagnose, and/or care for patients with dementia, $40 \%$ refer patients suspected of having dementia to another clinician. These patients are usually referred to neurologists (78\%), psychiatrists $(17 \%)$, and geriatricians $(16 \%)$. Verification of di-
Table 3. Physician Responses to Survey Items Related to Care of Patients with Dementia and Perceived Hard to Manage Phenomena

\begin{tabular}{lc}
\hline & "Yes" Responses, \\
Treatment and Care Item & $771(91 \%)$ \\
\hline "Do you provide routine ongoing primary & \\
$\quad$ care for patients with dementia?" & \\
$\quad$ n $=85$ lwere asked) & \\
Hard to manage dementia-related & \\
$\quad$ behaviors and complications & \\
(n = 771 responses)* & \\
1. Aggressiveness & $579(75)$ \\
2. Restlessness/agitation & $493(64)$ \\
3. Paranoia & $452(59)$ \\
4. Wandering & $436(57)$ \\
5. Sun-downing & $329(43)$ \\
6. Insomnia & $229(30)$ \\
7. Depression & $185(24)$ \\
Comorbidities associated with dementia & \\
that are hard to manage(n $=771)^{\dagger}$ & \\
1. Falls & $515(67)$ \\
2. Delirium & $423(55)$ \\
3. Adverse reactions to medications & $346(45)$ \\
4. Urinary incontinence & $321(42)$ \\
5. Poor appetite & $304(39)$ \\
6. Weight loss & $289(37)$ \\
Family/caregiver problems associated with & \\
$\quad$ dementia (n = 771) & \\
1. Fatigue/exhaustion & $554(72)$ \\
2. Planning for institutional placement & $519(67)$ \\
3. Anger & $346(45)$ \\
4. Isolation & $296(38)$ \\
5. Depression & $218(28)$ \\
6. Need for information & $175(23)$ \\
\hline
\end{tabular}

*Number of above behaviors \& complications difficult to manage: mean, 3.51; standard deviation (SD), 1.93.

${ }^{\dagger}$ Number of above comorbidities difficult to manage: mean, 3.33; SD, 1.61. Mean (SD) adjusted for all items in a subgroup, 3.19 (2.00).

agnosis (59\%) and long-term comanagement (54\%) were identified as the leading reasons for referral. For physicians who do not refer to a clinical specialist $(n=486)$, the primary reason was feeling comfortable in diagnosing and treating dementia (85\%), increased expenses to the patient for referral (35\%), and lack of specialists/resources in the community (20\%).

\section{Bivariate and Multivariate Analysis}

The independent variables found to be statistically significant at the bivariate level with a given outcome variable were subsequently included in a sep- 
arate logistic regression analysis (Table 4). Of the 11 independent variables identified initially, 3 were found to be significant $(P \leq .20)$ with both "order brain imaging" and "order more sensitive test," whereas 6 were observed for "referral" at the bivariate level. When entered into the regression analysis, we found no significant predictors $(P>$ $.05)$ for "brain imaging" and only one (percentage of a physician's patient population $\geq 65$ years old) for the use of a "more sensitive test." For this latter outcome, the adjusted odds ratios (AOR) varied by physicians' patient population age category using patient category of " $>40 \% 65$ years of age and older" as the referent group. These odds of conducting a more sensitive test for diagnoses are $40 \%$ lower (AOR, 0.60; $P=.20$ ), 53\% lower (AOR, $0.47 ; P=.007$ ), and $45 \%$ lower (AOR, $0.55 ; P=$ .03 ) for doctors with proportions of patients $\geq 65$ years old of $<5 \%, 5 \%$ to $20 \%$, and $21 \%$ to $40 \%$ respectively. Overall, the AORs ranged from 0.47 to 0.60 , suggesting that the odds of assessing pa- tients with a more sensitive test were generally lower for physicians reporting a smaller proportion of older patients compared with physicians with the largest proportion of older patients.

For the item "refer patients . . . to other clinicians," 3 items had statistically significantly correlation with this item. As the percentage of physicians' older patient population decreased, their odds of referral to other clinicians increased. Relative to clinicians with $\geq 40 \%$ of patients $\geq 65$ years old, the odds increased by $319 \%$ for proportions $<5 \%$ (AOR, 4.19; $P<.001$ ), by $194 \%$ for proportions between $5 \%$ and $20 \%$ (AOR, 2.94; $P<.001$ ), and by $54 \%$ for proportions between $21 \%$ and $40 \%$ (AOR, 1.57; $P=.07$ ). Rural physicians' odds of referral (yes/no) were 44\% lower than the odds for urban physicians (AOR, 0.56; $P=.009$ ). As the number of tools physicians perceived they needed to better screen/diagnose and treat patients with dementia increased, so too did their odds of referral (AOR, 1.13; $P=.04$ ).

Table 4. Results from Logistic Regression Analyses

\begin{tabular}{|c|c|c|c|}
\hline Outcomes and Predictor Variables* & $\begin{array}{l}\text { Adjusted } \\
\text { Odds Ratio }\end{array}$ & $\begin{array}{c}\text { Wald } \\
\chi^{2}\end{array}$ & $\begin{array}{c}P \\
\left(\chi^{2} \text { test }\right)\end{array}$ \\
\hline \multicolumn{4}{|l|}{$\begin{array}{l}\text { A. Order brain image as part screening or diagnostic evaluation for dementia } \\
\text { (yes/no) }\end{array}$} \\
\hline $\begin{array}{l}\text { Family/caregiver problems }(n=0-7) \text { regarding dementia endorsed by } \\
\text { respondent }\end{array}$ & 1.09 & 2.47 & .12 \\
\hline Professional time spent in direct patient care & 1.01 & 3.06 & .08 \\
\hline Time since medical school graduation (years) & 1.01 & 1.08 & .30 \\
\hline \multicolumn{4}{|l|}{$\begin{array}{l}\text { B. Assess positively screened patients } \geq 65 \text { years old with more sensitive tests } \\
\text { (yes vs. no) }\end{array}$} \\
\hline$<5 \%$ vs. $>40 \%$ & 0.60 & 1.62 & .20 \\
\hline $5-20 \%$ vs. $>40 \%$ & 0.47 & 7.33 & .007 \\
\hline $21-40 \%$ vs. $>40 \%$ & 0.55 & 4.60 & .03 \\
\hline Comorbidities $(n=0-6)$ associated with dementia endorsed by respondent & 0.94 & 1.09 & .30 \\
\hline $\begin{array}{l}\text { Tools }(\mathrm{n}=0-6) \text { needed to diagnose/treat dementia endorsed by } \\
\text { respondent }\end{array}$ & 0.92 & 1.58 & .21 \\
\hline \multicolumn{4}{|l|}{$\begin{array}{l}\text { C. Refer patients } \geq 65 \text { years old with suspected dementia to other clinicians } \\
\text { (yes vs. no) }\end{array}$} \\
\hline$<5 \%$ vs. $>40 \%$ & 4.19 & 16.89 & $<.001$ \\
\hline $5-20 \%$ vs. $>40 \%$ & 2.94 & 19.05 & $<.001$ \\
\hline $21-40 \%$ vs. $>40 \%$ & 1.57 & 3.29 & .07 \\
\hline Rural geographic location (vs. urban) & 0.56 & 6.77 & .009 \\
\hline Suburban geographic location (vs. urban) & 0.84 & 0.72 & .40 \\
\hline Female sex (vs. male) & 1.31 & 2.56 & .11 \\
\hline Dementia behaviors/complications $(\mathrm{n}=0-7)$ endorsed by respondent & 0.10 & 0.002 & .10 \\
\hline Comorbidities $(n=0-6)$ associated with dementia endorsed by respondent & 1.11 & 3.19 & .07 \\
\hline $\begin{array}{l}\text { Tools }(\mathrm{n}=0-5) \text { needed to diagnose/treat dementia endorsed by } \\
\text { respondent }\end{array}$ & 1.13 & 4.15 & .04 \\
\hline
\end{tabular}

*Independent variables included within each model above are those found to be statistically significant $(P \leq .20)$ at the bivariate level with the given outcome. There were 11 independent variables for each outcome at the bivariate level. 


\section{Discussion}

To our knowledge, this is the first national and systematic description of US family physicians' self-reported practice patterns focusing on the assessment and care of patients with dementia, although one qualitative study reported challenges of dementia care among Canadian family physicians. ${ }^{11}$ Among surveyed physicians whose patient populations include older adults (ie, those $\geq 65$ years old), an overwhelming majority (91\%) provide ongoing primary care for their patients with dementia. Moreover, $93 \%$ of these physicians also involve themselves in the screening and/or diagnostic evaluation for dementia of their older adult patients, although screening of asymptomatic patients is much less likely to occur compared with diagnostic evaluations (56\% vs. $90 \%$ ). Moreover, only a small percentage of physicians report that they only screen. These data are in line with the evaluation of evidence on screening for dementia by the US Preventive Services Task Force, which because of insufficient evidence provided no recommendations for or against routine screening in older adults. ${ }^{12}$

Of physicians who screen and/or conduct diagnostic evaluations, $73 \%$ report conducting a more sensitive test in their offices to confirm the presence of dementia. Unfortunately, the tests most commonly reported to be used are not particularly effective at identifying early dementia. More effective tools are now available, such as the Montreal Cognitive Assessment. ${ }^{13,14}$

At the time of this survey no physicians reported using web-based, computer-adapted tests. Computer-adapted tests have been demonstrated to have improved sensitivity and specificity in study settings compared with paper-based tests, ${ }^{15}$ but with little reason to make an early diagnosis, primary care clinicians have not adopted these tests in routine care. Should a disease-modifying therapy be discovered, the pressure for earlier diagnosis may change. As part of their evaluation of dementia, responding clinicians routinely collect patient history and order lab work, which is supported by current recommendations. ${ }^{16}$ Clinicians were less likely to order brain imaging (66\%); yet this testing rate may still be high, given that routine brain imaging in the diagnosis and treatment of dementia rarely leads to a change in therapy without the presence of focal neurological signs. ${ }^{17}$
Less than half of family physicians who care for dementia patients routinely refer these patients to other clinicians (40\%). Overall, responding family physicians are involved in all phases of assessment and routine care for patients suspected/diagnosed with dementia. It is noteworthy that only $4 \%$ of physicians with older-adult patients are not involved in screening, diagnosis, or routine care, despite the recognized problems surveyed physicians encounter in the assessment and care process, including relatively poor diagnostic tests ${ }^{18}$; few instruments to track the course of the disease; complex dementia-related behaviors (aggressiveness, restlessness, paranoia, wandering); comorbidities (falls, delirium, adverse medication reactions); and family/caregiver problems (fatigue, planning for institutional placement of patient with dementia). Given all these concerns, clinicians and their staff may find it difficult to successfully diagnose and manage patients with dementia without sufficient interdisciplinary support systems, education, and necessary training in routine clinical care. Recent evidence points out that the complex and multifaceted issues of identifying and providing care for patients with dementia in primary care are effectively addressed by comprehensive and coordinated care approaches. ${ }^{19}$ Some of the key elements for these comprehensive care models identified are the use of multidisciplinary teams of clinicians working closely with care coordinators, social workers, and community services; these elements are also part of patient-centered medical homes. As more practices transform into patient-centered medical homes, these services may be more widely available for caring for patients with dementia. The details on key enablers of successful care for patients for dementia have been presented elsewhere. ${ }^{9}$

Another area for future work is understanding how screening approaches could be successfully implemented in primary care, even though they are not indicated at this time. Even for proven successful care processes (eg, 9-item patient health questionnaire and asthma control test), widespread adoption can be difficult and long coming in busy primary care practices. ${ }^{20-23}$ The timeline to widespread adoption will likely be longer when a proven screening strategy has not even been developed. Perhaps because of the limited treatment options available for individuals with dementia and the US Preventive Services Task Force's indeterminate rating related to screening, improving dementia 
care across primary care practices seems to be receiving little attention at this time. Developing proven approaches to care would seem worth the attention of researchers and practicing clinicians. Effective approaches to the case-finding of symptomatic dementia patients in primary care are being developed. The application of the principles of the patient-centered medical home has been demonstrated to be effective ${ }^{24,25}$ in many chronic diseases and may provide added staff for diagnosis and care management, including behavioral health staff for caregiver stresses.

\section{Limitations}

We recognize several limitations to this research. First, our sampling frame was US AAFP members who spent at least $50 \%$ of their professional time in direct patient care and may not reflect the practice patterns of primary care clinicians in general or of family physicians who are not AAFP members. Second, we obtained a nonresponse rate of $40 \%$, despite 3 mailings. Obviously, we have no way of knowing how the nonrespondents might have affected the obtained results. While respondents had selected demographics statistically similar to those of the larger AAFP member population, representativeness across demographic factors does not necessarily equate with similarity in survey response patterns and vice versa. ${ }^{26}$ Third, the most important reason found for refusal to complete a survey is lack of interest in the subject. ${ }^{27}$ Therefore, family physicians who completed the survey were likely to be more interested in and knowledgeable about the assessment and care of patients with dementia. Fourth, a major concern related to survey research focusing on health care is that there may not be a close connection between what clinicians "say they do" and what they "actually do." ${ }^{28}$ We did not observe the actual practice patterns of the surveyed physicians. Thus, our estimates of family physicians who screen using one tool or another may be inflated.

Furthermore, the survey items developed for this project were not assessed for their psychometric properties (reliability and validity) before their use. For at least some items, respondents may not have shared the same interpretation of wording, resulting in different intended responses compared with the investigators' (and our physician consultants') interpretation of the responses. The instrument was piloted before use without any evidence of respondent misinterpretation, but piloting cannot always guarantee interpretation across a wider population. Moreover, some items could have been explored further to obtain more precise data. For example, the patterns of diagnostic evaluations adopted by participating physicians, similar to the description of the screening tools reported, could have been explored further. This would have given us a more complete picture of these practice patterns, although it would add to the length of the survey, likely resulting in a lower response rate. ${ }^{29}$

Finally, these data were gathered in 2008 and thus may not accurately reflect current screening, diagnostic, and care patterns typically found in primary care offices. For instance, the instruments used by those who screen/diagnose dementia may have changed, especially for use in the early detection of dementia. We observed that the primary tool used for screening was the Mini-Mental Status Examination among those physicians who reported screening.

\section{Conclusion}

The screening, diagnosis, and ongoing treatment of at least some patients with dementia seem to be expectations of the majority of family physicians practicing in the United States. Diagnostic instruments in routine use have relatively poor sensitivity and specificity, and instruments to monitor interventions are greatly needed. The aging of the US population along with the possibility of a treatment breakthrough suggest that dementia may warrant increased attention by the primary care community.

\section{References}

1. Alzheimer's Association. 2012 Alzheimer's disease facts and figures. Alzheimers Dement 2012;8: 131-68.

2. Plassman BL, Langa KM, Fisher GG, et al. Prevalence of dementia in the United States: the aging, demographics, and memory study. Neuroepidemiology 2007;29:125-32.

3. Hurd MD, Martorell P, Delavande A, Mullen KJ, Langa KM. Monetary costs of dementia in the United States. New Engl J Med 2013;368:1326-34.

4. Suehs BT, Davis CD, Alvir J, et al. The clinical and economic burden of newly diagnosed Alzheimer's disease in a medicare advantage population. Am J Alzheimers Dis Other Deme. 2013;28:384-92.

5. Chodosh J, Petitti DB, Elliott M, et al. Physician recognition of cognitive impairment: evaluating the 
need for improvement. J Am Geriatr Soc 2004;52: 1051-9.

6. Iliffe S, Robinson L, Brayne C, et al. Primary care and dementia: 1. diagnosis, screening and disclosure. Int J Geriatr Psychiatry 2009;24:895-901.

7. Valcour VG, Masaki KH, Curb JD, Blanchette PL. The detection of dementia in the primary care setting. Arch Intern Med 2000;160:2964-8.

8. Koch T, Iliffe S, project E-E. Rapid appraisal of barriers to the diagnosis and management of patients with dementia in primary care: a systematic review. BMC Fam Pract 2010;11:52.

9. Aminzadeh F, Molnar FJ, Dalziel WB, Ayotte D. A review of barriers and enablers to diagnosis and management of persons with dementia in primary care. Can Geriatrics J 2012;15:85-94.

10. Ferri CP, Prince M, Brayne C, et al. Global prevalence of dementia: a Delphi consensus study. Lancet 2005;366:2112-7.

11. Pimlott NJ, Persaud M, Drummond N, et al. Family physicians and dementia in Canada: Part 2. Understanding the challenges of dementia care. Can Fam Physician 2009;55:508-9.e1-7.

12. Boustani M, Peterson B, Hanson L, Harris R, Lohr KN; U.S. Preventive Services Task Force. Screening for dementia in primary care: a summary of the evidence for the U.S. Preventive Services Task Force. Ann Intern Med 2003;138:927-37.

13. Nasreddine ZS, Phillips NA, Bedirian V, et al. The Montreal Cognitive Assessment, MoCA: a brief screening tool for mild cognitive impairment. J Am Geritr Soc 2005;53:695-9.

14. Brandt J, Sullivan C, Burrell LE 2nd, Rogerson M, Anderson A. Internet-based screening for dementia risk. PloS One 2013;8:e57476.

15. Wild K, Howieson D, Webbe F, Seelye A, Kaye J. Status of computerized cognitive testing in aging: a systematic review. Alzheimers Demen 2008;4: 428-37.

16. Cordell CB, Borson S, Boustani M, et al. Alzheimer's Association recommendations for operationalizing the detection of cognitive impairment during the Medicare Annual Wellness Visit in a primary care setting. Alzheimers Demen 2013;9:141-50.

17. Albert M, DeCarli C, DeKosky S, et al. The use of MRI and PET for clinical diagnosis of dementia and investigation of cognitive impairment: a consensus report. Alzheimer's Association Neuroimaging Work Group Consensus Report; 2005. Available from: http:// www.alz.org/national/documents/imaging_consensus_ report.pdf. Accessed November 6, 2013.
18. Hatfield CF, Dudas RB, Dening T. Diagnostic tools for dementia. Maturitas 2009;63:181-5.

19. Karlin BE, Visnic S, Shealy McGee J, Teri L. Results From the Multisite Implementation of STARVA: a multicomponent psychosocial intervention for managing challenging dementia-related behaviors of veterans. Psychol Serv 2013 Aug 12. [Epub ahead of print]

20. Wittkampf K, van Ravesteijn H, Baas K, et al. The accuracy of Patient Health Questionnaire-9 in detecting depression and measuring depression severity in high-risk groups in primary care. Gen Hosp Psychiatry 2009;31:451-9.

21. Baas KD, Wittkampf KA, van Weert HC, et al. Screening for depression in high-risk groups: prospective cohort study in general practice. Br J Psychiatry 2009;194:399-403.

22. Wittkampf KA, Naeije L, Schene AH, Huyser J, van Weert HC. Diagnostic accuracy of the mood module of the Patient Health Questionnaire: a systematic review. Gen Hops Psychiatry 2007;29:388-95.

23. Ahmed S, Ernst P, Tamblyn R, Colman N. Validation of the 30 Second Asthma Test as a measure of asthma control. Can Respir J 2007;14:105-9.

24. Reuben DB, Roth C, Kamberg C, Wenger NS. Restructuring primary care practices to manage geriatric syndromes: the ACOVE-2 intervention. J Am Geriatr Soc. 2003;51:1787-93.

25. Melis RJ, van Eijken MI, Teerenstra S, et al. A randomized study of a multidisciplinary program to intervene on geriatric syndromes in vulnerable older people who live at home (Dutch EASYcare Study). J Gerontol A Biol Sci Med Sci 2008;63:283-90.

26. Galliher JM, Bonham AJ, Dickinson LM, Staton EW, Pace WD. Representativeness of PBRN physician practice patterns and related beliefs: the case of the AAFP National Research Network. Ann Fam Med 2009;7:547-54.

27. Cranford JA, McCabe SE, Boyd CJ, et al. Reasons for nonresponse in a web-based survey of alcohol involvement among first-year college students. Addict Behav 2008;33:206-10.

28. Montano DE, Phillips WR. Cancer screening by primary care physicians: a comparison of rates obtained from physician self-report, patient survey, and chart audit. Am J Public Gealth 1995;85:795-800.

29. Jepson C, Asch DA, Hershey JC, Ubel PA. In a mailed physician survey, questionnaire length had a threshold effect on response rate. J Clin Epidemiol 2005;58:103-5. 